\title{
U⿱宀⿻三丨口
}

\section{RFID in the production line for work flow improvement}

Curran, K., McClintock, A., Young, C., McKeag, D., \& Killeen, G. (2013). RFID in the production line for work flow improvement. International Journal of Agile Systems and Management, 6(1), 83-112. https://doi.org/10.1504/IJASM.2013.052226

Link to publication record in Ulster University Research Portal

\section{Published in:}

International Journal of Agile Systems and Management

\section{Publication Status:}

Published (in print/issue): 11/03/2013

DOI:

10.1504/IJASM.2013.052226

\section{Document Version}

Author Accepted version

\section{General rights}

Copyright for the publications made accessible via Ulster University's Research Portal is retained by the author(s) and / or other copyright owners and it is a condition of accessing these publications that users recognise and abide by the legal requirements associated with these rights.

\section{Take down policy}

The Research Portal is Ulster University's institutional repository that provides access to Ulster's research outputs. Every effort has been made to ensure that content in the Research Portal does not infringe any person's rights, or applicable UK laws. If you discover content in the Research Portal that you believe breaches copyright or violates any law, please contact pure-support@ulster.ac.uk. 


\title{
RFID in the production line for work flow improvement
}

\section{Kevin Curran* and Andrew McClintock}

School of Computing and Intelligent Systems,

University of Ulster,

Northland Road, Londonderry, BT48 7JL, N. Ireland

E-mail: kj.curran@ulster.ac.uk

E-mail: mcclintock-a@email.ulster.ac.uk

Corresponding author

\section{Charles Young}

NuPrint Ltd,

Springtown, Derry, BT48 9HH, Northern Ireland

E-mail: charlieayoung@hotmail.com

\section{Dennis McKeag}

Faculty of Computing and Engineering,

University of Ulster,

Jordanstown, Co. Antrim, BT52 1SA, Northern Ireland

E-mail: dennismckeag@btinternet.com

\section{Gavin Killeen}

NuPrint Ltd,

Springtown, Derry, BT48 9HH, Northern Ireland

E-mail: gavin@nuprintuk.com

\begin{abstract}
Radio frequency identification (RFID) technology is becoming more embedded in the supply chain. Tracking goods in the work place has been identified as one area where production can be improved. In fact, in almost all non-'green field' modern manufacturing facilities, there are work flow process systems that can be improved to reduce the overall work load and increase the total work throughput. This paper outlines a 'real life' manufacturing facility where RFID was integrated to help stream line work flow.
\end{abstract}

Keywords: radio frequency identification; RFID; lean manufacturing; location awareness; indoor tracking; localisation.

Reference to this paper should be made as follows: Curran, K., McClintock, A., Young, C., McKeag, D. and Killeen, G. (2013) 'RFID in the production line for work flow improvement', Int. J. Agile Systems and Management, Vol. 6, No. 1, pp.83-112. 
Biographical notes: Kevin Curran is a Reader in Computer Science at the University of Ulster. His achievements include winning and managing UK and European framework projects and technology transfer schemes. He is a regular contributor to $\mathrm{BBC}$ radio and TV news in the UK and has produced over 700 publications.

Andrew McClintock holds a first class honours degree in Electronic Engineering from the University of Ulster. He is currently employed in the Northern Ireland IT industry and his research interests include RFID, production systems and programming.

Charles Young holds a first class honours degree and a $\mathrm{PhD}$ in Engineering from the University of Ulster. He obtained a scholarship from AVX in Coleraine for the BEng Hons Engineering degree. His $\mathrm{PhD}$ was sponsored by Rolls-Royce and involved modelling carbon fibre composites for the aircraft turbine engine fan blades. He was the Knowledge Transfer Process (KTP) Associate on the project detailed in this paper.

Dennis McKeag is a Professor Emeritus of Product Development at the University of Ulster. For the past 25 years, he has specialised in product and process innovation through knowledge and technology transfer programmes between academia and industry, and is an acknowledged leader in this field. He has over 100 publications and other forms of public output, and has refereed papers in a number of conferences and publications in his areas of specialisation.

Gavin Killeen holds a BA in Business Studies from the University of Ulster. He is the Managing Director of Nuprint Ltd. which supplies labels to major food, drink and beverage companies. He has maintained its investors in people status, achieved ISO 9001:2000 UKAS and in 2006 achieved the BRC/IoP food packaging high standard which is also UKAS product certified. He is the Chair of the North West Workforce Development Forum, a DENI appointee on the Board of Governors of Foyle and Londonderry College, a board member of Young Enterprise North-West and is the Vice-President Elect of the Londonderry Rotary Club.

\section{Introduction}

It is often said in the automation industry that to control, you must first measure. Radio frequency identification (RFID) is a non-contact, long distance, water proof, high temperature resistant, data storage, automatic identification system. These attributes make RFID the ideal solution for tracking and measuring the flow of physical items throughout a plant. The RFID system comprises of an integrated collection of components, the tag, the reader, the reader antenna, a controller, a sensor, actuator and annunciator (optional), host and software system and communication infrastructure (Lahiri, 2005). These qualities allow RFID to play an important role in allowing the physical flow of equipment throughout a plant to be linked to, or create an information flow that is real-time. This type of information used in the correct way can allow for a transparent plant wide view of how the plant runs, enabling users to see and predict bottlenecks 
and backlogs. In acquiring this data, the plant is then in a position to allow for data interrogation in order to optimise plant or process activities. Lean Manufacturing is a process of data interrogation in order to eliminate any non-value adding tasks (NVAT) thus improving efficiency (Wong et al., 2009).

All manufacturing facilities must possess and adhere to their own manufacturing systems. These systems are the foundation on which any industrial accreditations are built. They define the work flow process and are therefore critical to all aspects of how the company operates. The tools the systems are built on usually refer to two types, electronic tools, such as software packages and written documents manually created, maintained and archived. A vast majority of these systems operating today have been developed onsite by skilled employees knowing their own responsibility and therefore produced a tool that delivers what his or her department needs to. These tools have been developed in a similar fashion, as quality controls or accreditation standards increase or the business changes. It is therefore accepted that a vast majority of these tools certainly serve their purpose, but are not as efficient or as transparent as they could be.

In today's global manufacturing environment the western world is at a disadvantage because of high labour costs, high land rates, stringent environmental rules and regulations. These factors make it difficult for manufacturing facilities to compete with its neighbouring Asian counterpart. It is no surprise then that our manufacturing industry is in a consolidation period and looking to maximising efficiency by increasing utilisation of their current assets. This is clearly reflected in industrial management buzzwords. These buzz words are encapsulated by two ideas, Lean manufacturing and Total Cost of Ownership. Lean Manufacturing was a system originally developed by Toyota and defines wastefulness as any activity that is non-value adding. It was claimed by implementing lean manufacturing, you can use less of everything compared to mass production- half the human effort in the factory, half the manufacturing space, half the investment in tools, and half the engineering hours to develop a new product. In addition, it requires keeping less than half of the needed inventory on site, results in a lot fewer defects, and produces a greater and ever-growing variety of products. In short, it is called lean because it uses less, or the minimum of everything required to produce a product or perform a service (Wong et al., 2009).

All this can be achieved by reducing NVAT's at every stage in the system. Total cost of ownership analysis then looks at the total cost of the system for its life time. These costs include but are not limited to, cost of installation, preventative maintenance, corrective maintenance, operational costs, repair costs and end of life costs and expand the buyer's thought process beyond the initial purchase cost (Ritsma et al., 2009). Therefore for manufacturing facilities to address the problem areas they must first find them, thus the solution requires gathering more data, gathering the data quicker, making the data more transparent and easy to access, increase communication ability and increase tractability. In comparing RFID technology to the traditional barcode system to provide the technology for the solution it has many advantages including but not limited to, short scan times, anti pollution and durable, flexible data, penetrability through other materials, usable user data and better security (Ohashi et al., 2008). Given these advantages it is easy to see why the technology has been adopted in a wide range of industries such as logistic, Healthcare, toll systems, retail, security and identification to name a few. This project will look to integrating RFID into the current 
manufacturing work flow system to expose these advantages thus reducing waste and the TCO.

The 'real life' facility is NuPrint Technologies LTD, a local manufacturing company who manufacture labels. NuPrint management have highlighted that there is an opportunity to reduce waste in two key production stages. These stages are known as pre-prep and production. Pre-prep, as the name suggests prepares the equipment before use in production, and maintains it after the job is complete. The equipment is in the form of plates and rollers. The plates must be wrapped around the roller and aligned. Production fit the plates to automated printing machines and produces the required label. The pre-prep process is not as straight forward as it may seem. This is due to a multiple of variables including, damaged or weakened plates, plates being difficult to align, prioritisation of batch jobs, sourcing and reserving common plates to multiple jobs as well as keeping in touch with Operation control and Production to continually evaluate the job status.

The aim of this project is to integrate an RFID solution into a manufacturing execution system (MES). It is hoped that the RFID solution will allow the MES to view the process in greater detail in terms of job and equipment location in real time. The real time MES will provide a transparent view of the process to Pre-prep, production and operation control. This electronic view will allow each department to have up to date process information with no need to ask the other department, thus greatly reducing the requirement for personnel interaction. Personnel interaction is a major fluctuating unknown, which can be very wasteful. The real time view will also build a history database of the information gathered, as well as having the ability to hold any additional notes the operators may want to add.

\section{RFID and lean manufacturing}

The focus of this project is to integrate automated tracking technology into a factory in order to streamline the production phase. As a consequence, we provide a background here to the key aspects of the project. As previously discussed every manufacturing facility has manufacturing systems in place to define the work flow process, these systems are usually referred to as MES. In general these systems are open loop systems or at best a one shot production feed back to indicate the status or whereabouts of a particular job. This one shot feedback is usually in the form of the operator confirming their task is completed and ready for the next stage or department. Although this information is valid and useful, it is somewhat too late, i.e.,, if department one enters that it is finished with job one, department two will in many cases already know because job one will be in their department. Figure 1 illustrates this in practice.

So the question is what can be done better? The answer is more interaction at multiple steps within each department. The argument against this is 'same solution same result' as the previous, not to mention the added work load it would give to the department. It is therefore clear that RFID with its automatic detection provides the answer to allow greater data input to an MES without burdening the department. 
Figure 1 Time frame of one shot feed back (see online version for colours)

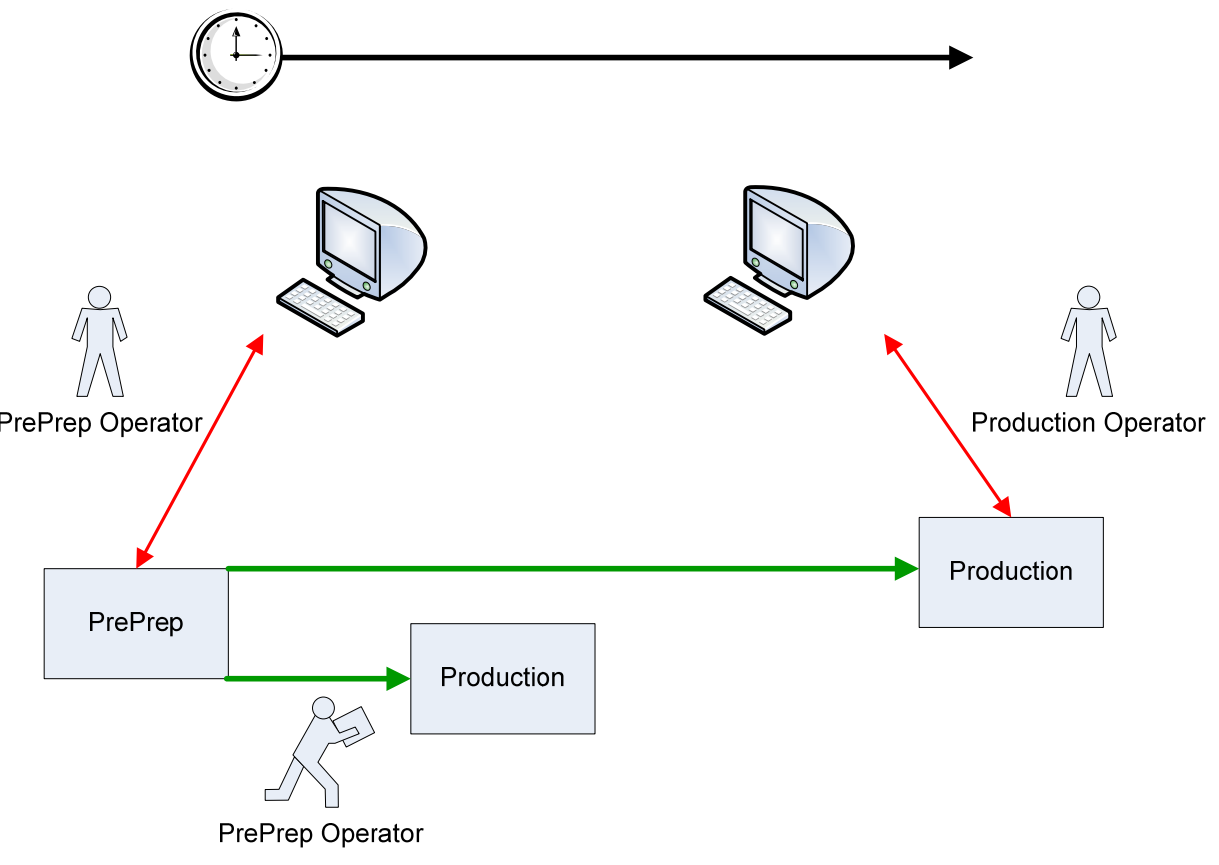

At this stage it is important to evaluate this technology. Here we will review how and where this technology has been implemented in similar settings to NuPrint and evaluate the advantages and disadvantages of the solution. The Department of Industrial Engineering, Tsinghua University, Beijing, setup a micro plant as to best simulate a real plant situation. RFID technology was used to support the MES to automate the tracking of materials, work in progress (WIP), fixed and mobile resources. Table 1 shows where the RFID equipment was located and the data it was able to provide the MES.

Table 1 Deployment of RFID devices

\begin{tabular}{ll}
\hline Objects & Deployment of RFID readers and tags \\
\hline Materials and WIP's & $\begin{array}{l}\text { Stick tags to their containers (pallets, bins and boxes, etc) } \\
\text { Workers }\end{array}$ \\
Eacilities and conveyors & $\begin{array}{l}\text { Deploy RFID readers to the shelves in the warehouse and the sorting } \\
\text { centre, the sorting tunnel in the sorting centre, the work benches in the } \\
\text { assembly line, the material reservation boxes by workstations, and } \\
\text { thevehicles conveying materials and products, the ceiling of the } \\
\text { laboratory for tracking workers from above. }\end{array}$ \\
Allocate portable readers for material checking
\end{tabular}

They designed and implemented the solution to target three key areas. These were data collection and document control, labour management and production control and performance analysis. Data collection and documentation control is a continuous task throughout the manufacturing process, but are of particular advantage in the job 
scheduling and inventory control. The best example of this advantage comes at the beginning of the manufacturing system, after a customer places an order, an operator scans the RFID labelled inventory stock, this real time scan, allows the MES to decide if the required inventory is available to complete the customer request. If it is, the job is sent to the sorting centre for packaging before being sent to the assembly line. However, if the required inventory is not available the MES system automatically generates a purchase order (PO) and produces it for review before e-mailing it to the supplier.

Labour management and production control in this solution were encapsulated by visibility. Two billboards where utilised, one for the assembly line operator which updated them on the current job information and segment procedure. After the job is completed the operator interfaced with the MES system and indicated if it was finished or scrapped, triggering a job status change. The second billboard allowed management visibility of the production schedule being completed, including the current status of jobs. Performance Analysis is achieved as a byproduct of the previous two implementations. The system now is data rich, and is used to target key performance indicator (KPI) matrices such as work station load and production efficiency. Any target change made can now be evaluated on tangible numeric data that is non-intrusive (Chen et al., 2009).

RFID MES system can be flexible and responsive to continuous, changing customer requirements (Huang et al., 2010). They outline an RFID MES implementation in JAC, an automotive production company based in china. JAC manufacture a full line of brand vehicles including trucks, special reconnaissance vehicle's (SRV's) and mechanically propelled vehicle's (MPV's). JAC already had an MES system using traditional data acquisition methods such as manual input and barcodes. However, they believed the gap between physical flow of product and information flow in the MES was too great to properly monitor and manage the production system. The additional data acquisition obtained by attaching RFID tags to tools, materials, personnel and equipment ensured the MES turned into a real time MES (RT-MES). The enhanced RT-MES decreased SRV cycle time from five days to four days, increased production efficiency by $20 \%$ and increased the MES data accuracy rate to $99.9 \%$. It is important to look at what the technology has in store for the future. One key area for future development is combined logistical tracking with RFID and GPS (He et al., 2009). The advantage here is the ability to link both business process info and geographical locations, this specifically targets the time consumed for sales, customer service, operation and warehouse staff to locate specific cargo in transit and provide the customer with the most accurate data in a time frame that is acceptable. This data could also be shared between manufacturers, logistics and purchasers on the web so that better planning and scheduling can be done for incoming inventory.

For this project, it is important to note that the solution was purely a prototype and some known issues would still need to be resolved before this type of solution would be made viable. The two biggest issues being, integration at both hardware and middleware level as well as data privacy (He et al., 2009).

As mostly all the research has shown positive results for the application it is important that negatives are actively looked for in order to clearly grasp the technology. The known limitations are poor performance with RF-opaque and RF-absorbent objects, Impacted by environmental factors, limitations on actual tag reads, Impacted by hardware interference and limited penetration power of the RF energy. RF-opaque materials such as metal and RF-Absorbent objects such as water can cause issues with the RFID technology resulting in partial or failed reads. RF-Opaque materials cause the radio signal 
to bounced or reflect, causing the reader to receive multiple reads or incomplete reads. This is known as multipath and can cause reliability issues especially when the surrounding materials are constantly changing. Environmental Factors, such as rain, human traffic, vehicle traffic, electrically noisy equipment and existing older wireless LAN's on the $900 \mathrm{MHz}$ range all can cause interference for the RFID system. Actual tag reads are limited. The reader employs a type of anti collision algorithm to identify tags; therefore the reader has to interact with the tag several times before the tags information can be confirmed. This extra communication limits the readable amount of tags per second. This limit differs greatly between manufacturers but is steadily on the increase. Hardware Interference is seen when two reader's signals overlap causing data collision. This is usually seen on poor installation and usually easily avoided. Penetration power is determined by transmitted power of the reader. This power level determines the signal strength of the reader and therefore directly proportional to the distance and penetration levels. This power level is determined by individual country laws and therefore not universal (Lahiri, 2005).

\subsection{Lean manufacturing}

Toyota is widely credited with devising Lean Manufacturing. Toyota created a work flow process called Toyota Production System (TPS) or Just in Time (JIT). The TPS system was developed between 1949 and 1975 and was an amalgamation of the Ford production system and other techniques. Shingo and Ohno refined these techniques taking into consideration the up and coming labour unions and with a different outlook on factory workers giving them more ownership resulting in better team development and cellular manufacturing. However, the single biggest refinement from the Ford Production came from the need for the system to be flexible. The Ford system was designed for a single, never changing product. TPS reduced setup times to minutes which allowed for small batches but resembled a continuous flow allowing for greater flexibility within each small batch (New, 2007). As Toyota started to reap the rewards of it manufacturing system, like minded Japan and American businesses tried to learn and implement the system with mixed results. Some of the reasons being the lack of understanding of the underlying principles of TPS, or the system was not integrated into the complete system. The system was relabelled Lean manufacturing by Womack et al. (1990).

Today Lean's accepted fundamental element is to reduce waste by producing only what is needed when it is needed. In doing this the desired outcome is to reduce lead time and cost, improve quality and increase productivity. This principle is broadly accepted and so, has been adopted into many manufacturing disciplines. The single biggest down side of this, is that Lean seems to have many faces and interpretations, it is not a single set of guidelines that can be rolled out across any industrial environment, in fact the complete opposite, each situation must be evaluated on its own merits and implemented as such to achieve the end goal. In taking the fundamental principle of Lean to 'reduce waste' we must ask what the types of waste are. Again, lean breaks these down into seven important areas which are overproduction, excess inventory, over processing or incorrect processing, waiting, unnecessary transport or conveyance, unnecessary movement and defects. However clear the fundamental principle and the types of waste; it is clear that the areas to practice are not so. We have seen how the Lean system has changed between Ford Production to TPS to allow more flexibility for employees. This continues when safety in the workplace was at the forefront and even today where people management 
and culture is at the forefront, this is easily seen when comparing different approaches to practice for the lean manufacturing system (Wong et al., 2009) concluded that there are 14 key areas to assess waste. These areas are:

- Work processes - standardisation allows better quality control and less job deviation, resulting in better time management.

- Scheduling - adoption of the 'pull' mentality and method helps to control inventory both coming in and out.

- Inventory - controlled to a minimum, thus reducing roof space required as well as reducing depreciation value on stock.

- Equipment - uptime and availability of equipment is critical to the plant operations so any activity such as preventative maintenance to ensure when equipment is needed it is ready will greatly improve plant performance.

- Layout-determines the flow of parts or equipment throughout the plant. The closer each location is to where it is receiving and sending stock the better.

- Material handling - key to reducing waiting times and damage to goods in transit.

- Employees - empowered and motivated employees are the best tool any facility can have and critical to all aspects of the business.

- Quality - essential in reducing waste in the truest meaning of the term.

- Product design - producing the required quality of product with the cheapest materials.

- Suppliers - suppliers with the JIT mentality help reduce need for inventory stock.

- Tools and techniques - aids to implement and control examples of such tools are 5S, Steam mapping, poka-yoke, lean 101 and cellular flow to name a few.

- Customers - relationships and understandings allow both parties to meet each other's needs as well as predict demands

- Ergonomics and safety - the foundation of all activities, keep employee morale and available work time to a maximum.

- Management and culture - critical in implementing and supporting any lean activities.

An example of how Lean Manufacturing methodology and principles help in a real world case is documented in (Willhite, 2004). They claimed that their traditional work system encouraged large batch sizes and high inventory all to accommodate the 'push' mentality of both them and their customers. Facility analysis included identifying non-value added tasks (NVAT) and value added tasks (VAT) using the Value stream mapping tool. The mapping out of the current physical work flow throughout the factory floor and the redesign of a more stream line layout was devised; this was accompanied with the $5 \mathrm{~S}$ tool to allow minimum obstructions and distractions. They also reduced batch sizes by $35 \%$, reduced and standardised batch time by documentation and operation standardisation, and turned process flow bottlenecks in to supermarket pull systems. Visual aids throughout the facility also kept facility KPI's at the forefront of everyone's mind to help in employee motivation and moral. As a result of this implementation, on time delivery has 
increased to $100 \%$, inventory is now controlled by demand, labour capacity has increased and all excess work has been eliminated (Willhite, 2004).

In conclusion, there are key areas for reflection. The first of which is RFID's ability to become the eyes and ears of the MES. The technology is at a point now of maturity, this maturity brings with it a host of advantages including mass information and technical data, multiple manufacturing vendors and the ever lowering cost to purchase. Another key area is the MES system. This is the information processing system that will allow for quality data to be gathered. The system must be data rich, with accurate real time data. Entering data into the system must not be an extra work burden on the department. The system must be intuitive and flexible in its ability to produce reports to target NuPrints KPI's. Employees implementing the solution and adopting the solution must be clear and motivated to the end goal, with the understanding that one of the keys is continuous improvement. This continuous improvement inevitably means the first solution may not always be the best but more a base on which to improve. There is no single recipe for successful implementation. Each facility is unique and in turn requires a unique solution. The best solution will be gained by firstly fully detailing the end result, fully analysing what is currently happening and targeting the most wasteful areas that hinder the end result to find a solution that can reduce the waste. After implementation the solution must again be analysed and further improvements made to continuously improve the solution until an optimum is reached.

\section{Requirement analysis}

The client for this project was a local label manufacturing facility in Northern Ireland. The requirement is to incorporate RFID technology into a new or existing MES to alleviate repeated paperwork, reduce the data recorded, automatically assign responsibilities, reduce material wastage, identify potential holdups and generally increase/improve communication. The MES will address the management, manufacturing and purchasing departments. In order to get the most from any solution it must be applied in the worst affected area. This can only be done by fully analysing the current facility and how physical work flow and data flow is monitored and captured. As an introduction to this, each department is detailed with its own duties and responsibilities.

The front office is where the where the interaction between NuPrint and its customers and suppliers happen. All customer requests are made directly to the front office as well as raw material purchases. The operations control department manages storage, production and pre-prep. It is the central point of decision making. Operations control must evaluate each managed departments schedule, inventory and resources and make production decisions. The Pre-prep area stores, prepares and maintains the printing plates. Plates are a type of stencil that are approximately the size of an A4 page, they are made of a flexible rubber. Each plate applies only one colour; there can be up to a maximum of seven colours or plates per label. The plates must be mounted on a roller and aligned. Once this is completed the plates are ready for production. When production is finished with the mounted plates, pre-prep dismount the plates, clean them and put them in uniquely marked plate files for storage. Production runs the printer, this task includes mountings the printing plates, raw paper roll and ink trays to run the printer. Storage holds manufactured inventory until orders are complete, then dispatches to the customer. Storage also hold raw material inventory. 
Figure 2 shows how the facility is laid out and the typical personnel movement required to get a label batch produced from start to finish.

Figure 2 Nuprint facility layout (see online version for colours)

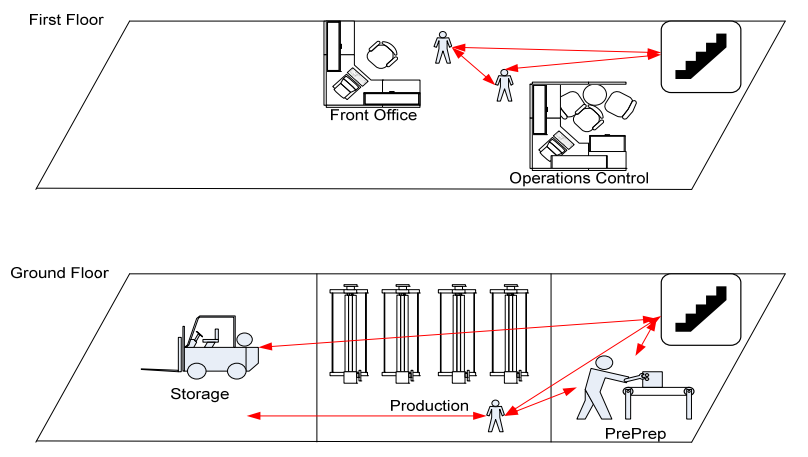

After initial analysis, it is clear from the personnel movement that there are areas of concern i.e., the work flow is not fluid and there is a lot of required points of contact for each department to allow them to complete their individual tasks. This gives ground for a detailed analysis of both physical and data flow for a complete label batch production. It is clear that the area of greatest concern in the system is the communications between pre-prep and the production area.

\subsection{Factory production issues}

Now that the target area has been highlighted it must be assessed what issue this causes the running of the current manufacturing system. The issues are outlined and explained below.

- Time: plate files are ordered/ numbered according to product codes; it is time consuming to locate plate files. Also, in differing jobs for the same customer, common plates occur and there place of location can be in any of that particular customer files, e.g., Nuprint may have five differing files dedicated for the same customer and one plate may be common and used for each of these jobs. This plate is usually situated in the file of the job last produced and so must be located for each of the 4 remaining jobs. Locating this plate requires research into the last job ran for that customer which is time consuming - particularly if the plates have not been washed, placed back in the file and returned to the rack.

- Records: records regarding file usage are written manually, this is not only time consuming but open to operator error.

- Aesthetics: stickers indicating if plates have been damaged and replaced are located on the file exterior which is untidy and confusing

- Traceability: knowledge of plate preparation or printing status can only be established by intervening/chasing up. There is no record in terms of plate usage, date, job number and the machine; who located, mounted, removed, washed or last 
stored the plates; difficulties associated with plate mounting and the total metres the plates may have printed since purchase

- Individual issues: a typical example of an individual issue was, during a period when the pre-prep operator was off ill the pre-prep operators duties fell on the production operators. However, the process of washing the plates is an undesired task coupled with the limited time the production operator had, it was decided the plates would be left unwashed in a pile and not cleaned or filed away. The consequence of this was that when an urgent job came through, a delay was incurred in locating the plates. When the plates were located a further delay was incurred because they had to be washed and mounted. Once the plates were placed on the machine and the job 'setup' ready for 'signoff', it transpired that two were damaged as they had been situated at the bottom of the pile and were subsequently compressed. This necessitated the re-purchase of two $£ 70$ plates at a $£ 10$ delivery cost. The operations control was also faced with the dilemma of cleaning down the machine to run a job while the replacement plates were being manufactured and delivered or to hold off on the wash-up/setup and run the job the next day. This expense was in addition to delaying delivery of the labels. The result of this bad relationship was an escapade wasting $£ 100$ 's and damaged reputation with a customer.

Table 2 Business evaluation

\begin{tabular}{|c|c|c|c|c|c|}
\hline Production to preprep & $\begin{array}{l}\text { Occasions } \\
\text { asked }\end{array}$ & $\begin{array}{l}\text { Time taken to } \\
\text { ask/answer } \\
\text { (min) }\end{array}$ & $\begin{array}{c}\text { Total time taken } \\
\text { to ask/answer } \\
\text { (min) }\end{array}$ & Risk & $\begin{array}{c}\text { Actual } \\
\text { time } \\
\text { (Pre) }\end{array}$ \\
\hline Where are you & 2 & 2 & 4 & 1.1 & 4.4 \\
\hline What is the next job & 1 & 2 & 2 & 1 & 2 \\
\hline Do you have the required plates & 1 & 1 & 1 & 1.2 & 1.2 \\
\hline Condition of plates & 1 & 2 & 2 & 1.3 & 2.6 \\
\hline Are common plates involved & 1 & 0.5 & 0.5 & 1 & 0.5 \\
\hline $\begin{array}{l}\text { Problems associated with } \\
\text { mounting the plates }\end{array}$ & 2 & 3 & 6 & 1.3 & 7.8 \\
\hline What is holding you up & 1 & 1 & 1 & 1 & 1 \\
\hline Why is the place so messy & 1 & 1 & 1 & 1 & 1 \\
\hline $\begin{array}{l}\text { Who washed and mounted } \\
\text { plates }\end{array}$ & 1 & 3 & 3 & 1.2 & 3.6 \\
\hline \multicolumn{6}{|l|}{ Preprep to production } \\
\hline Where are you & 2 & 2 & 4 & 1.1 & 4.4 \\
\hline When do you need the plates & 1 & 2 & 2 & 1 & 2 \\
\hline $\begin{array}{l}\text { When will the cylinders be } \\
\text { available }\end{array}$ & 2 & 1 & 2 & 1.1 & 2.2 \\
\hline $\begin{array}{l}\text { Do you remember issues with } \\
\text { job }\end{array}$ & 1 & 2 & 2 & 1.2 & 2.4 \\
\hline What's holding you up & 1 & 1 & 1 & 1 & 1 \\
\hline Why is the place so messy & 1 & 1 & 1 & 1 & 1 \\
\hline $\begin{array}{l}\text { Time taken to Ask/Answer } \\
\text { (min) }\end{array}$ & & & 32.5 & & \\
\hline $\begin{array}{l}\text { Actual time taken pre solution } \\
\text { installation }\end{array}$ & & & & & 37.1 \\
\hline
\end{tabular}




\subsection{Evaluation}

In order to see the effects this has on the business it is important to equate the waste accumulated in this stage of the process. Table 2 breaks down the questions asked between production and pre-prep, into the time taken to ask and answer, it goes further to evaluate the risk of each question i.e., how often it is conceivable that the given time would adequately answer the question. Risk $=1+$ the probability the time allocated is sufficient.

This data must now be equated to financial waste. From the above data, we can see that the dispersion of the total waste 37.1 minutes is broken down into $24.1 \mathrm{~min}$ for Production and 13 min for pre-prep. Assuming that NuPrint run 1.5 batches a day these times increase to $36.1 \mathrm{~min}$ for production and 19.5 for pre-prep. Taking a pre-prep value at $£ 30.00$ per hour, the waste can be calculated as:

Financial waste $=($ Waste Time $(\min ) / 60) \times 30=(19.5 / 60) \times 30=£ 9.75$ per day

Taking a production value at $£ 120.00$ per hour, the waste can be calculated as:

Financial waste $=($ Waste Time $(\min ) / 60) \times 120=(36.1 / 60) \times 120=£ 72.2$ per day

This equates to a total of $£ 81.95$ of financial waste per day.

Figure 3 System architect overview (see online version for colours)

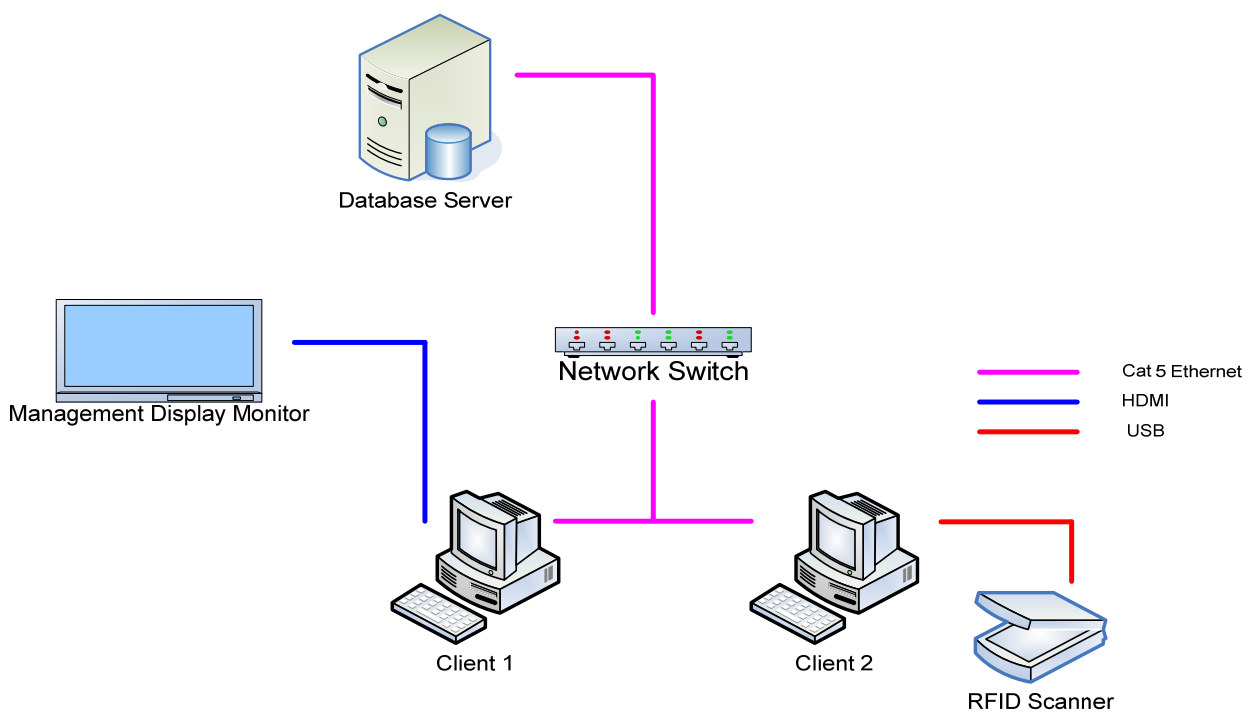

\section{RFID system design}

The design of this solution is to implement RFID in a manufacturing environment, to aid in the stream lining of production work flow. The incorporation of RFID itself may not achieve this, but using RFID as a data gathering tool to enrich databases with real-time information can target important areas to the business. These areas of the business 
include real time information which will highlight work flow bottlenecks. Monitoring this information during and after an operational fix has been implemented to reduce the bottleneck which will allow management to evaluate the fix and continue to improve it or move on to the next high priority bottleneck. In addition, operational procedures are stream lined whereby operatives must complete the previous task before moving on to the next stage. Figure 3 shows the conceptual overview of the system. The database server provides data storage and manipulation; clients provide operator and management interfaces, RFID scanner provides real-time data input, while the management display monitor provides a clear operational view to high level management.

Figure 4 System communications (see online version for colours)

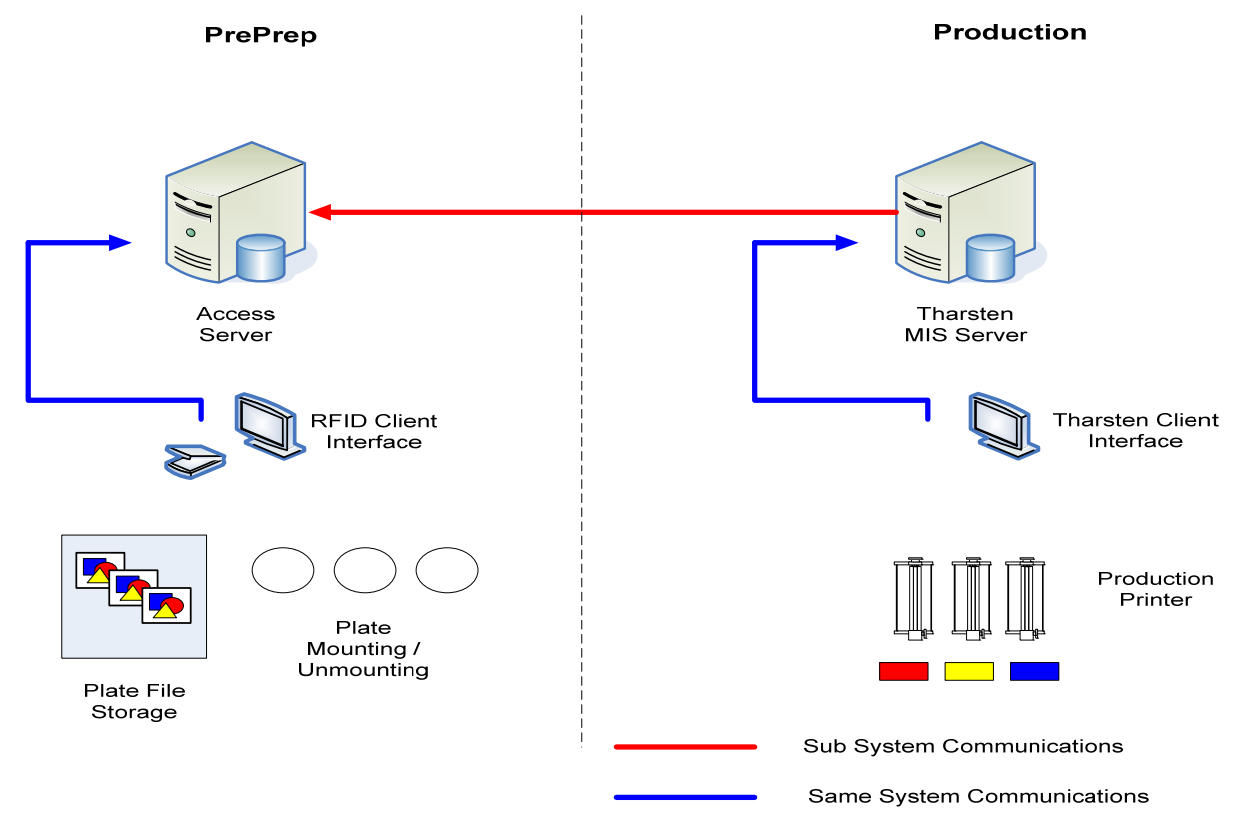

\subsection{Management information system integration}

During the design of this solution,the client (Nuprint) bought an 'off the shelf' Manufacturing Information System (MIS) called Tharsten. Tharsten's MIS was implemented to control the production area and gathers a lot of critical information via user interfaces regarding the running of the printing presses. It was clear that if we could extract already gathered information from the Tharsten system we could greatly improve the richness of data in the Access database and therefore achieve better results. In light of this, Figure 4 shows a more detailed architectural overview of how data is gathered and shared between both systems. Data transfer is broken down into two types, same system communications and sub system communication. Same system communications is the server interacting with its own type of client to enable database entries, this takes place on both the Access side and the Tharsten side and is inherent in the systems and therefore relatively straight forward. What is not as usual is the passing of data between the systems, i.e., sub system communications. The Tharsten system is an SQL-based server 
and after some research and testing it was decided that the sub system communications could be reliably delivered via an Open Database Connectivity (ODBC) link, note during discussions with Tharsten technical engineers it was clear that to maintain the Tharsten system integrity and support we could only read data from their system with no function of writing data to their system.

Given financial waste outlined earlier, it is clear there is an opportunity to introduce a system that will aim to tackle this area of high waste. It was decided that the specification is to incorporate an RFID system to operate as follows. Firstly, there would be a scan in pre-prep to locate plate file and log time and confirm job is mounted ready for production and log time. Next, a scan at production to identify and acknowledge receipt thus logging time beginning job production and indicate job completion and log time and finally a scan in pre-prep to confirm receipt of plates and log time and to confirm plates have been cleaned, restored and log time. Figure 5 depicts where RFID scanning will be implemented and gives an overview of the final RFID setup.

Figure 5 RFID installation in factory (see online version for colours)

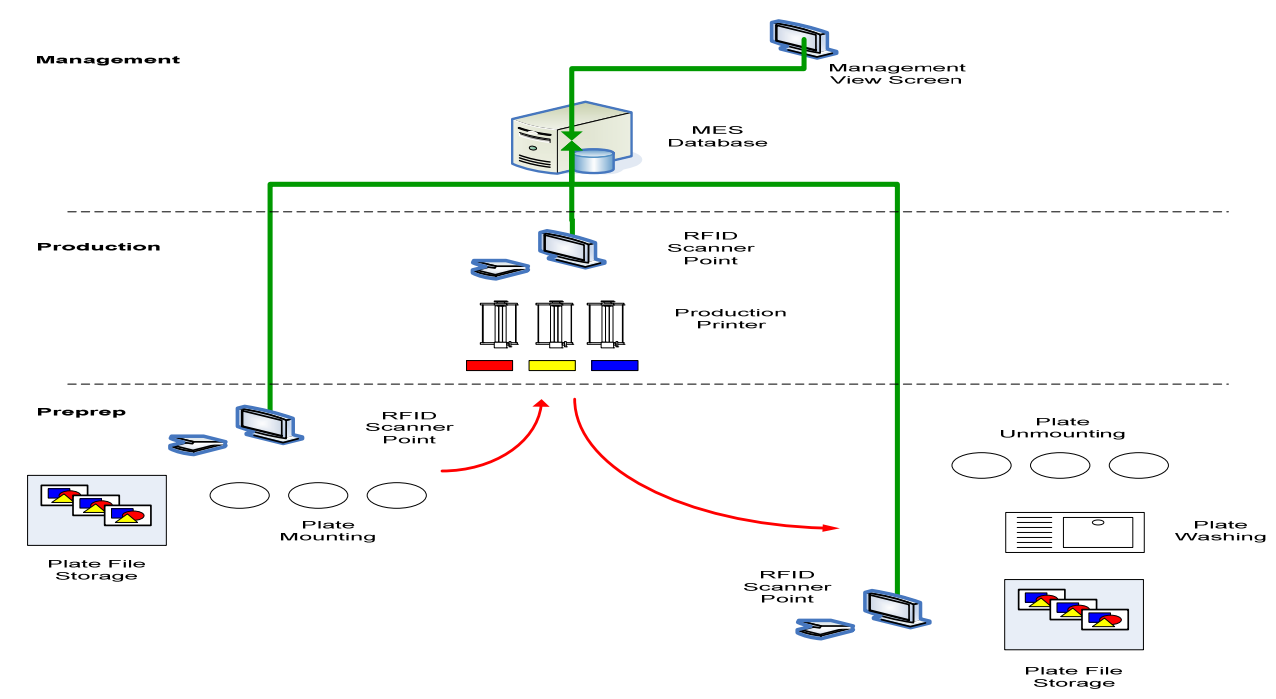

The incorporation of RFID should enable production control to establish the overall job status. These include the time taken to mount job, time spent in transit/waiting from pre-prep to the press, time spent on press and backlog of plates to be cleaned by pre-prep. The system should increase communication between production and pre-prep i.e., it should enable pre-prep to see the press status i.e., when the previous job has been scanned in by the printer to anticipate when a new job should be prepared. It should also allow pre-prep to see when the printing has finished and when the used plates/cylinders should be collected and production to check on the status of their next job. The system will provide traceability and accountability. It will enable JIT production with pre-prep preparing the next job only when the previous one has been scanned/received by the press. This will prevent wasted time caused by pre-prep mounting jobs too early in advance and potentially having to dismount them for an urgent job. 


\subsection{System operation}

After consultation with Nuprint and considering the new benefits the Tharsten system would have on the RFID system, the system operation was designed and a clear vision of how the final solution should interact with the operation steam. The complete system is now collecting critical manufacturing performance measuring parameters. These parameters are outlined in Table 3.

Table 3 Performance parameters

\begin{tabular}{lcccc}
\hline Job number & 19620 & 19621 & 19635 & 19622 \\
Customer & O'Kanes & Utterly Butterly & Tesco & Donegal C \\
No. of plates & 5 & 8 & 7 & 6 \\
Operative & Fiona Curran & Paul Lynch & Fiona Curran & Fiona Curran \\
Out of Hold Time & $27 / 01 / 2011$ & $27 / 01 / 2011$ & $26 / 01 / 2011$ & $27 / 01 / 2011$ \\
& $13: 15$ & $14: 05$ & $13: 00$ & $13: 00$ \\
Job Dispatch Time & $27 / 01 / 2011$ & $27 / 01 / 2011$ & $26 / 01 / 2011$ & $27 / 01 / 2011$ \\
& $13: 50$ & $14: 55$ & $15: 40$ & $14: 00$ \\
Production Start Time & $27 / 01 / 2011$ & $27 / 01 / 2011$ & $26 / 01 / 2011$ & \\
& $14: 20$ & $15: 05$ & $16: 10$ & \\
Production Stop Time & $28 / 01 / 2011$ & $28 / 01 / 2011$ & & \\
& $10: 30$ & $15: 30$ & & \\
Operative & Paul Lynch & Paul Lynch & & \\
Wash Start Time & $28 / 01 / 2011$ & $28 / 01 / 2011$ & & \\
& $15: 40$ & $15: 40$ & & \\
In Hold Time & $28 / 01 / 2011$ & $28 / 01 / 2011$ & & \\
& $15: 55$ & $16: 25$ & & \\
\hline
\end{tabular}

This array of data immediately brings clarity to operational performance. For example, we can see that operative Fiona Curran initially mounted and dispatched the O'Kanes job and then Paul Lynch then took over and mounted the Utterly Butterly job while O'Kanes was in production. Both jobs ran over night, then Paul Lynch signed on to Tharsten at 15:40 to begin washing plates and the time he put them into hold was recorded, Paul Lynch can see how long it took himself to wash plates according to the 'In hold' times, Fiona Curran mounted the Tesco job on the 26th and it is still on the press, Fiona began the Donegal $\mathrm{C}$ job but was delayed on the dispatch, she however completed the job before Paul took over. The Donegal $\mathrm{C}$ job is still waiting to go on the press and it took 15 minutes to wash plates 19620 and 30 minutes to wash plates 19621. In addition to this information, the system must also be capable of displaying any history associated with the plate file. Table 4 shows what these parameters should be.

Table 4 Plate file history parameters

\begin{tabular}{lccccccc}
\hline \multicolumn{7}{c}{ Plate file history parameters } \\
\hline $\begin{array}{l}\text { Previous } \\
\text { job no. }\end{array}$ & $\begin{array}{c}\text { Date when } \\
\text { previously ran }\end{array}$ & $\begin{array}{c}\text { Meters } \\
\text { printed }\end{array}$ & $\begin{array}{c}\text { Running } \\
\text { total }\end{array}$ & $\begin{array}{c}\text { Mounted } \\
\text { by }\end{array}$ & $\begin{array}{c}\text { Washed } \\
\text { by }\end{array}$ & Previous comments \\
\hline 19501 & $\begin{array}{c}21 / 09 / 2010 \\
11: 20\end{array}$ & 2,500 & 2,500 & RW & RW & $\begin{array}{c}\text { Plate 4 crosshairs } \\
\text { misaligned }\end{array}$ \\
19620 & $\begin{array}{c}27 / 01 / 2011 \\
14: 20\end{array}$ & 3,000 & 5,500 & FC & PL & $\begin{array}{c}\text { Same problem with } \\
\text { plate 4. Notified Laura }\end{array}$ \\
\hline
\end{tabular}


There are two important reasons for displaying these parameters to the operative. The first is to allow the operative a quick view of the total meters printed by the plates. Plates have a life span and do shatter when worn, the ability to view the meters printed by the plate already (running total) will allow the operative to call if the plates are good enough to go into production avoiding the current and likely case where plates shatter during production causing many manufacturing issues and wastes. The second reason is to allow the plate mounting operative to see if there is a history of any mounting issues (previous comments) associated with the plates. Plates can, at times be difficult to align on the roll and this will allow the operative to call if it would simply be more cost effective to order a new plate or consider if there is a quality concern with the plate supplier.

\subsection{RFID reader and integration}

The RFID reader came with its own supporting software to enable users to read RFID tags, however to ensure user interaction was kept to a minimum this functionality needed to be integrated into an Access form. The design of this was based around the mscomm control. Although the desktop reader is a USB device its internal software makes it appear as a serial connection to the machine. Mscomm control allows Access to take control of the machine's serial communication ports, set the communication protocol and transfer or read data from the connected device. During the design period it was also important to have a clear idea of the reader and tag capabilities in terms of scan able distances and amount of tags scanned at one time. The outcome of which would drive the procedure used when the operative would scan in and out the job from hold. Table 5 shows the results of tests specifically looking at these capabilities. From the results it is apparent that for reliability reasons tags will have to be read individually and be physically on the reader when scanned.

Table 5 Scanner and tag capabilities

\begin{tabular}{|c|c|c|}
\hline Label information & Type & HF $18 \times \times 18 \mathrm{~mm}$ wet inlay \\
\hline & Air interface protocol & IS015693 \\
\hline & Operation frequency & $1,365 \mathrm{MHz}$ \\
\hline & Frequency sector & High Frequency (HF) \\
\hline & Memory & $1 \mathrm{~K}$ bit \\
\hline \multirow[t]{10}{*}{ Reader testing } & Amount of labels read & Reliability \\
\hline & 1 label laid flat on the plate & $100 \%$ \\
\hline & 2 labels laid flat on the plate & $80 \%$ \\
\hline & 4 labels laid flat on the plate & $50 \%$ \\
\hline & 6 labels laid flat on the plate & $60 \%$ \\
\hline & Distance & Reliability \\
\hline & On the plate & $100 \%$ \\
\hline & $10 \mathrm{~mm}$ away from the plate & $90 \%$ \\
\hline & $15 \mathrm{~mm}$ away from the plate & $<10 \%$ \\
\hline & $>15 \mathrm{~mm}$ away from the plate & $0 \%$ \\
\hline
\end{tabular}




\subsection{Database design}

The implementation of the database must adhere to the principles under pinning good database design. The first of these principles is that redundant data (duplicate information) is not desirable, duplicate information significantly increases the likelihood of errors and inconsistencies and wastes space. The second principle is that the correctness and completeness of information is important. If the database contains incorrect information then all subsequent reports and queries will also be incorrect. The database divides information into subject-based tables, reducing redundant data. It provides each table with the information it requires to create tangible relationships. The Job number or UID is then used to achieve this. It also accommodates all queries. Figure 6 shows an overview of the database.

Figure 6 Database design overview (see online version for colours)
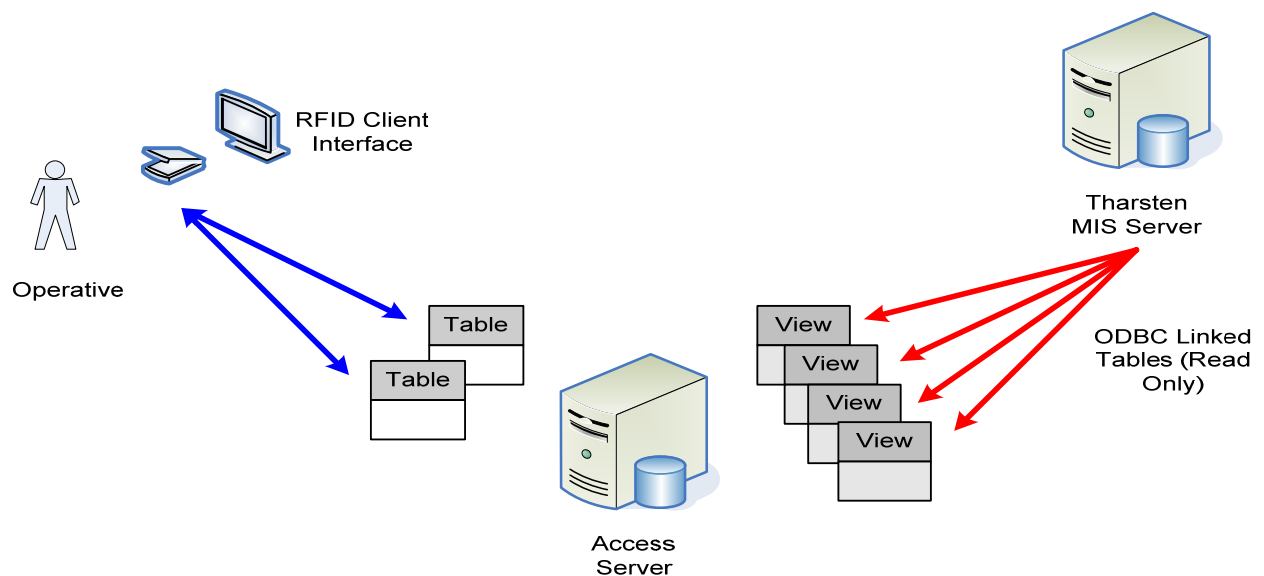

After the database is assembled relationships can be implemented to amalgamate data into usable structures.

\subsection{Form and query design}

After consultation with Nuprint it was clear that they required two main interfaces, one, to maintain their plate file database allowing the RFID labels to take over the old labelling system of Plate File numbers. The second interface is to allow production information to be inputted for the beginning and end of a job cycle in the Preprep area. These interfaces must be unambiguous, and simple to use. They must not increase the work load of operations and whether gathering information from Tharsten, RFID or Access appears to be one coherent system.

Interface one, the Nuprint RFID Plate File Record, is available to operations to associate RFID labels with the Plate File details. These details include, number of plates, Customer, supplier reference, number of colours, die size and product code. The interface will allow all relevant information to be shown with a simple scan button to associate a label with the plate file details. This interface will be used heavily on system implementation as Nuprint has approximately 350 current plate files, after this initial use 
the interface will only be used when a new job has been created for a customer or the RFID tag needs updating for an existing plate file.

Interface two, the Nuprint RFID Plate File Record, is the main operative interface. The interface is broken down into three main sections. Section one shows the operative interactions broken down into four main steps. Step one is for the operative to select an appropriate job, the choice available must only be jobs dispatched from Tharsten not yet picked up by Access, i.e., new jobs. Step twois to scan the plate file out of holding, there should be logical checks done in the background that ensures the correct plate file has been removed from holding by the operative, if the wrong one has been removed the operative should be prompted and not allowed to continue. Step threeis to associate a plate cleaning operative and time with the plates after production has taken place. Step four is the scanning of the plate file back into hold and thus completing the job cycle. All steps must be clear and concise as to the operative actions. Section two of the interface will display the active job status the details contained in this view is outlined in Table 3. Section three displays the plate file history of the current plate file the details contained in this view is outlined in Table 4. Figure 7 shows how the form was developed from early revisions to ensure user friendliness and additional work was kept to a minimum.

Figure 7 User friendly form design (see online version for colours)

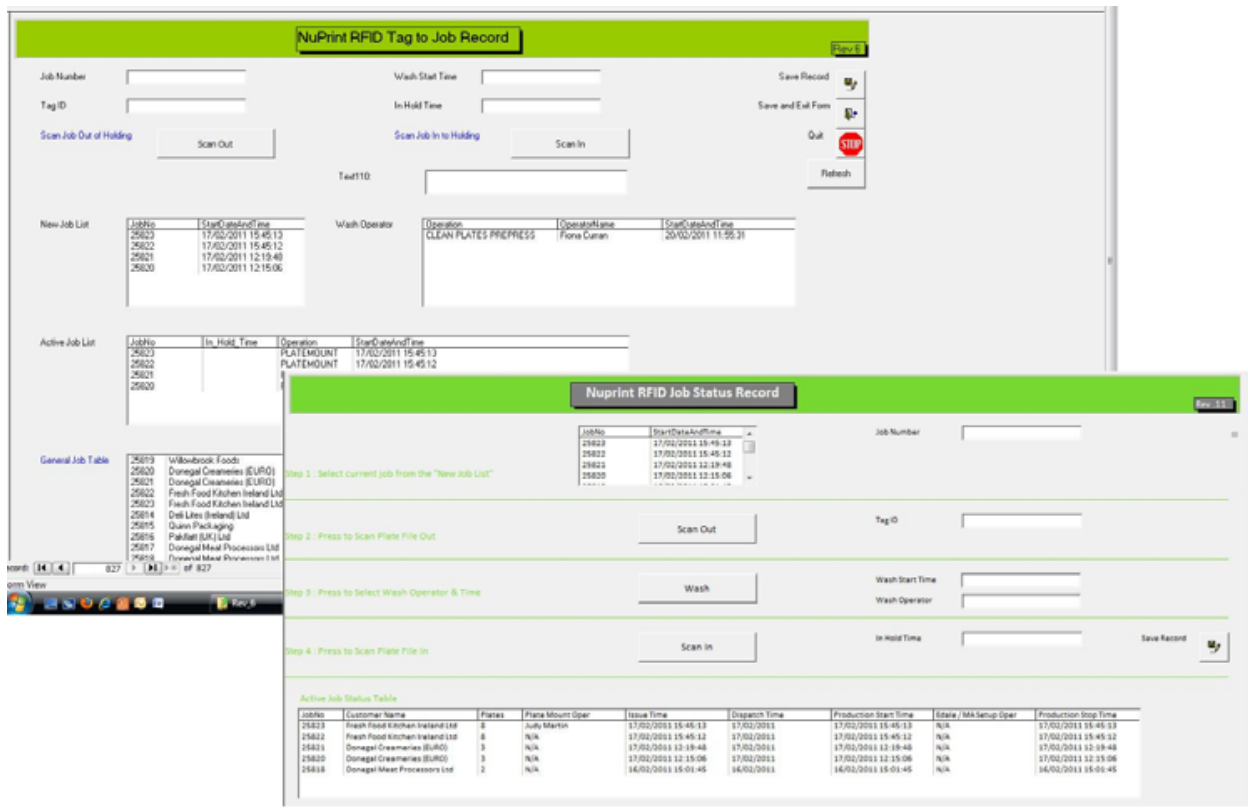

The solution required multiple queries to provide operations and management with quality data to help improve work flow. The outputs of which provide dispatched jobs not yet picked up by operations, active jobs currently being manufactured, number of plates required per job, dates and times of specific actions, operator responsible for specific actions and the accumulative meters a plate has produced and sub divided into the specific jobs. 


\section{RFID system implementation}

This section outlines the implementation required to achieve the goals outlined in the design section. It is decided that this section will familiarise key components and talking points of the document, then look at the end product, the two interfaces and work back into and thought them to see the database workings behind them. As a means of familiarisation the following images show key components referenced throughout this document.

Figure 8 Selection of RFID tags (see online version for colours)

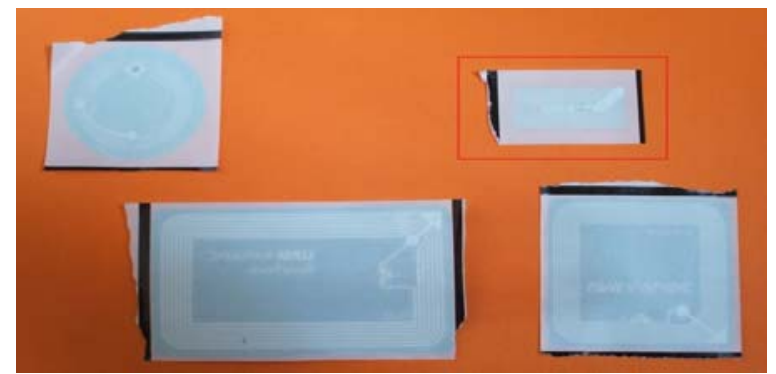

Figure 8 shows a selection of RFID tags purchased by Nuprint, the tag outlined in red is the tag used for implementation.

Figure 9 Nuprint plate file storage (see online version for colours)

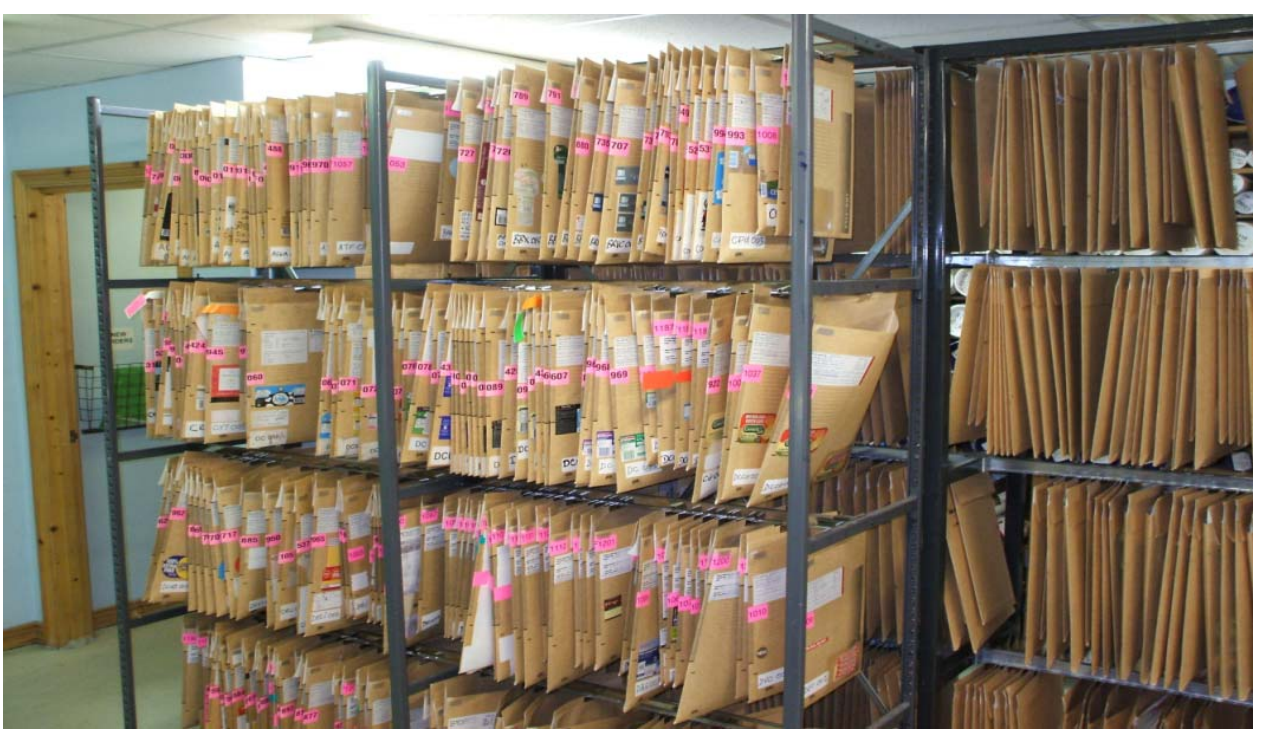

Figure 9 shows the plate file storage area, it highlights the volume of plate files and therefore how the organisation and upkeep of them can cause so many issues for Nuprint. 
Figure 10 Plate file details (see online version for colours)

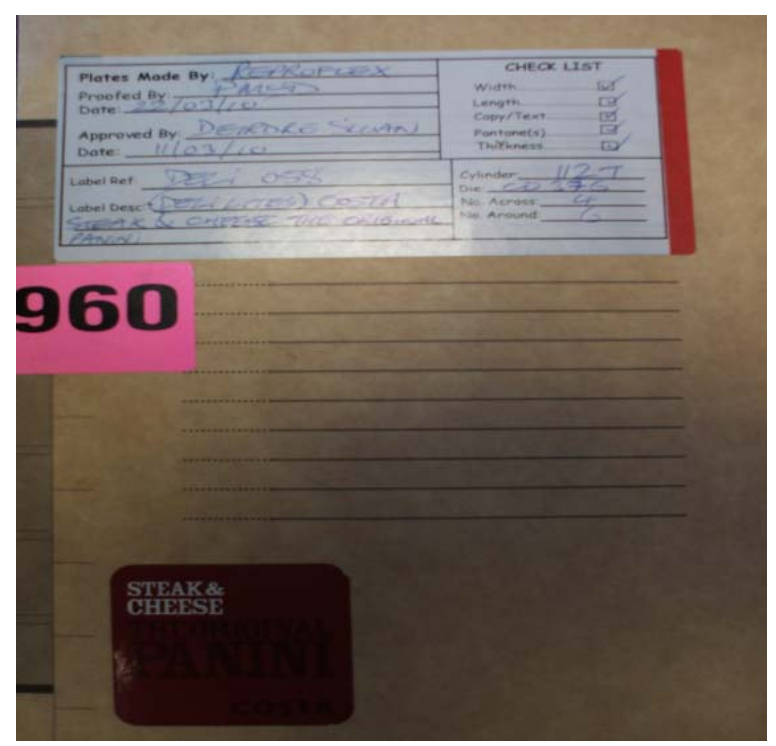

Figure 10 shows the current method of keeping the plate file record details. This highlights clearly how ambiguity in handwriting or plate files a few years old can lead to traceability issues.

Figure 11 Plate and mounting cylinder (see online version for colours)

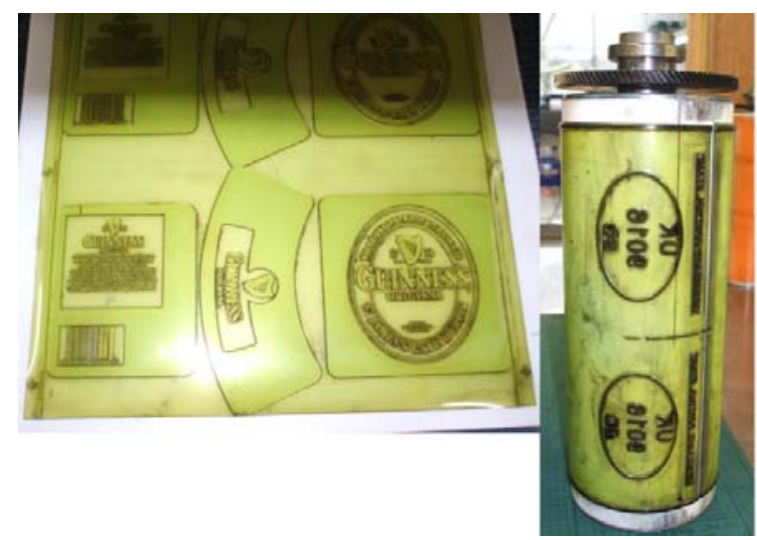

Figure 11 shows an individual plate file and how it is mounted onto a cylinder. This also gives an appreciation of how mounting and aligning can be difficult and important to document and flag when next running that particular job.

\subsection{RFID plate file record interface}

Figure 12 shows the Nuprint RFID plate file record interface where the operative would associate an RFID tag with the plate file and its details. The interface is laid out in three 
steps. Step one is to read the RFID tag, step two is to fill out the plate file details, finally in step three saving the data and searching for a new plate file to associate an RFID tag.

Figure 12 RFID plate file record interface (see online version for colours)

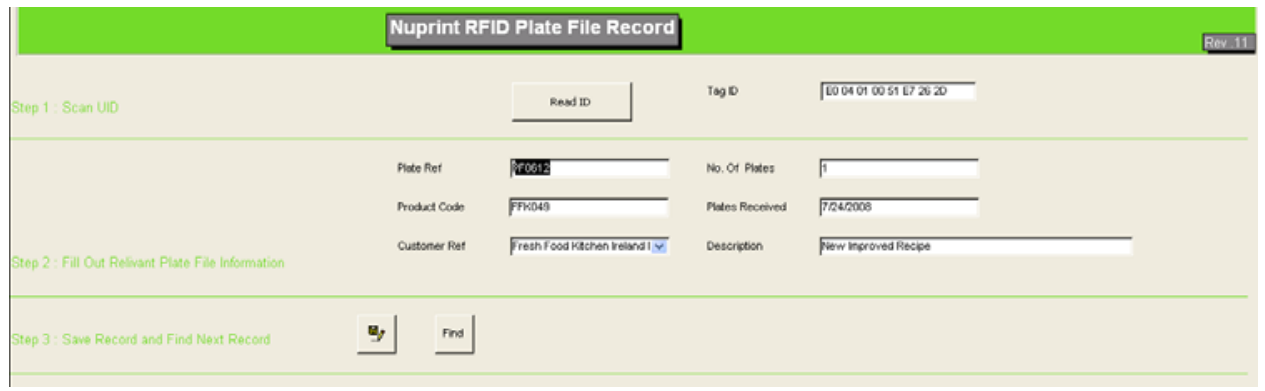

\subsection{RFID job status interface}

Figure 13 shows the second operative interface. This interface is designed to allow an operative to start and complete the full job cycle activities that occur in the Preprep area. As discussed in the design section, some of the data shown is gathered by the Tharsten system, this interface must allow for those relevant parameters to be viewed and allow additional Access database only parameters to be entered but presented as one coherent system.

Figure 13 Nuprint RFID job status record (see online version for colours)

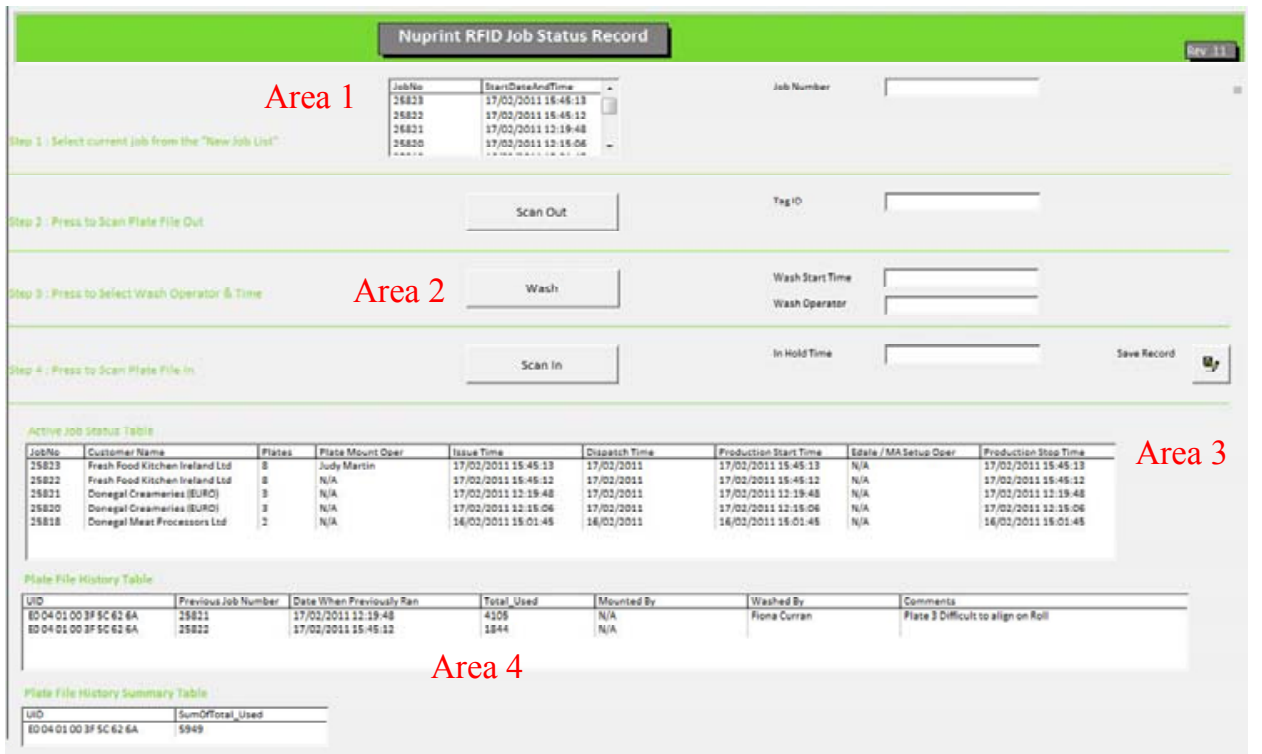

The critical information these tables do not hold is the Preprep area details. These details include a 'Wash Operator' 'Wash Start Time' 'In Hold Time' and associated 'Comments' all with reference to a 'UID' and 'Job Number'. With the correct placeholders now in 
place, queries can be created to show a limited view to the operative as to ensure they only select and see the information relevant to them. The first of these queries is the New Job List (New_Job_Query). A new job is defined as any job that has been created by Tharsten and sent to 'PLATEMOUNT' but has not been associated an RFID tag i.e., the plate file has not been taken out of hold.

1 Step 1 for the operative is to select the appropriate job from the query which in turn populates a bound text box and begins the Access side of job tracking.

2 Step 2 is to associate a plate file and therefore RFID tag with the job number. This is done with the same code as the Plate File Record interface, however there is a critical logical check also done at this stage when the RFID tag is read. This check is to ensure the Tharsten job description which always begins with the product code also held in the Access table Plates match up. This is a critical check, it ensures the operative has selected the correct plate file. The check is implemented by looking up both the Tharsten tables and Access tables and comparing, if it passes the check, inform the operative, if not, inform the operative and withdraw the scanned UID.

3 Step 3 is carried out after the job has been run on the press, this step is to associate anoperative, and time with the task of washing the plates. The operative has to sign into Tharsten under an activity of 'Clean Plates Prepress' however this is not job specific on the Thasten system. This brings a clear advantage to the Access system. The interface request the operative to select the 'Wash' button in Step 3 clearly identifying a particular operative with this task.

4 Step 4 is similar to Step 2 whereby the plate file is scanned, however during this step all that is required is to ensure the same plate file that was scanned out is being scanned in and associate a time with placing the plate file back into hold. If this check is passed the bound text box is populated with the current time, if not the operative is informed.

Area 3 of Figure 13 shows the Active Job Status table, an active job is defined as any job that has been created in Tharsten, associated a RFID but has not been returned to storage yet. This area is built on the Active Job Query. This table is of critical importance to the operative, as it allows them a quick view of how jobs are performing on the production floor. It will also catch a frequent situation at Nuprint whereby operatives take the plate file out of storage, run the job but don't wash up the plates and return them to storage. A quick glance at this table will allow visibility of jobs that went out a suspiciously long time ago and have not yet returned.

Area 4 of Figure 13 shows the plate fill history. This table is at first invisible to the operative, it becomes visible to the operative once they have scanned the plate file out of storage in Step 2. The table then displays critical data to allow the operative to make better informed decisions regarding the Preprep tasks.

\subsection{System walk through}

Given the form technical workings we will now look at how it is presented to the operative with a walk through of how it is to be used.The initial but less used interface is the 'Plates' form, the interface is only used when a new label has been designed and therefore requires a new set of printing plates. The Operative has only two main steps to 
complete, one to 'Read ID' where they are prompted with a message box if the scan has completed successfully or not. The second is manual input data on the plates and save the record. Figure 14 shows the form filled out with the message box prompt.

Figure 14 Operative view plates form (see online version for colours)

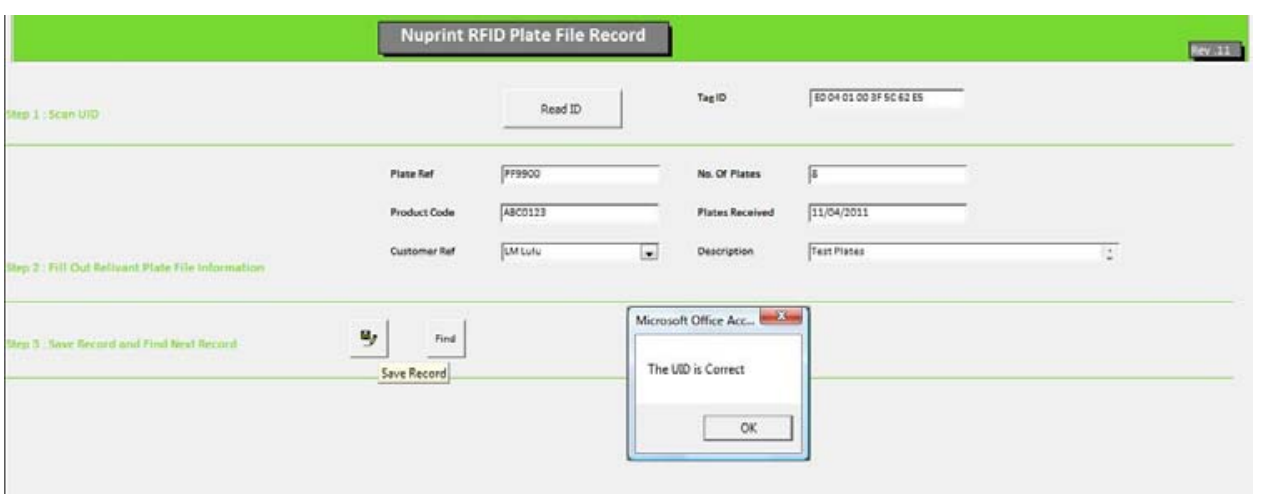

The main interface for daily use is the 'Jobs' form. This interface is used to initially assign a plate file to a job, monitor its progress in production via Tharsten and complete the job cycle by assigning the plate file back into storage after it has been successfully cleaned. Figure 15 shows what the operative is initially shown on loading the interface.

Figure 15 Operative view jobs form (see online version for colours)

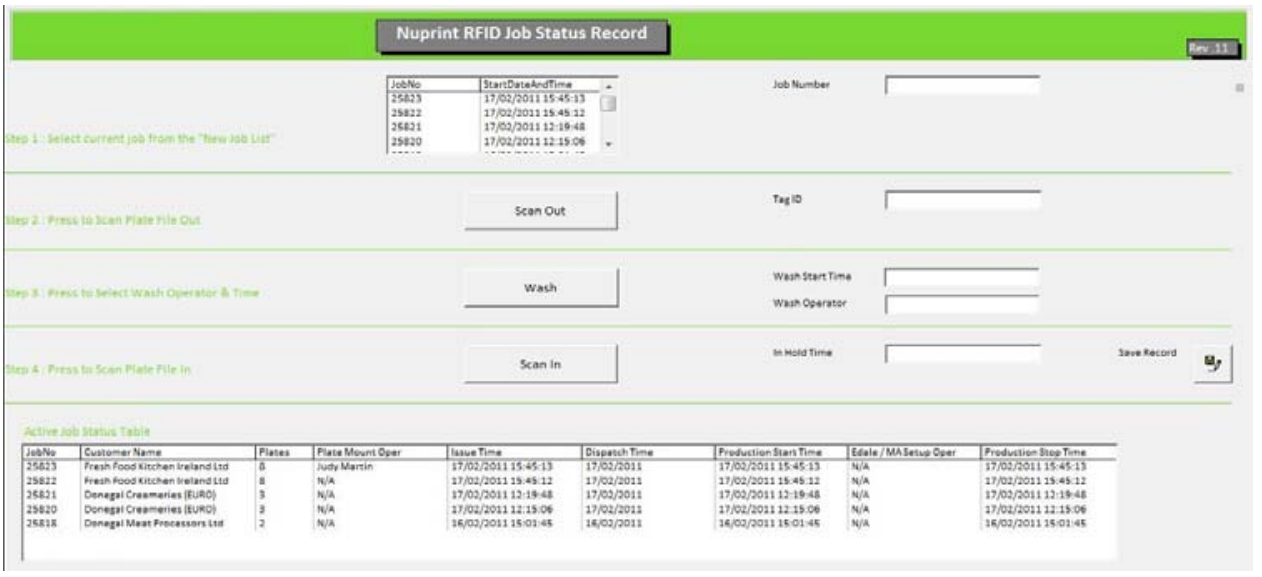

Step 1 is to select the appropriate new job from the new job list, this automatically populates the bound text.

Figure 16 Step 1 (see online version for colours)

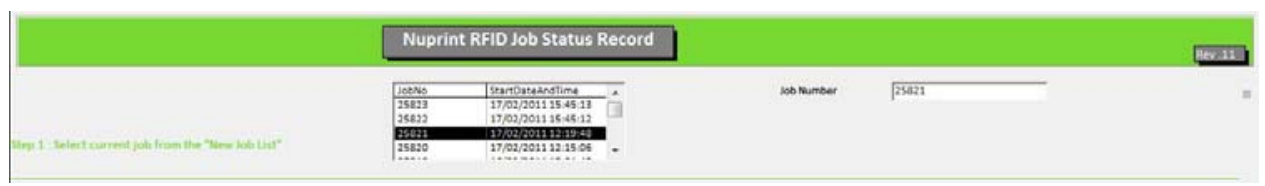


Step 2 is to scan the correct plate file out of storage, two stage logic is applied at this step, first the UID is checked to ensure it complies with the UID general structure, which results in a 'The UID is correct' message box, the second stage logic is to ensure the Product Code held in the Plates table (i.e., access table) is the beginning of the Tharsten job description (i.e., Tharsten parameter) this ensures the operative has opted to mount the correct plates, verified both by Tharsten and Access, this results in the message box 'The Plates Corresponds with the Tharsten job continue'. Figure 17 shows the resulting messages.

Figure 17 Step 2 (see online version for colours)

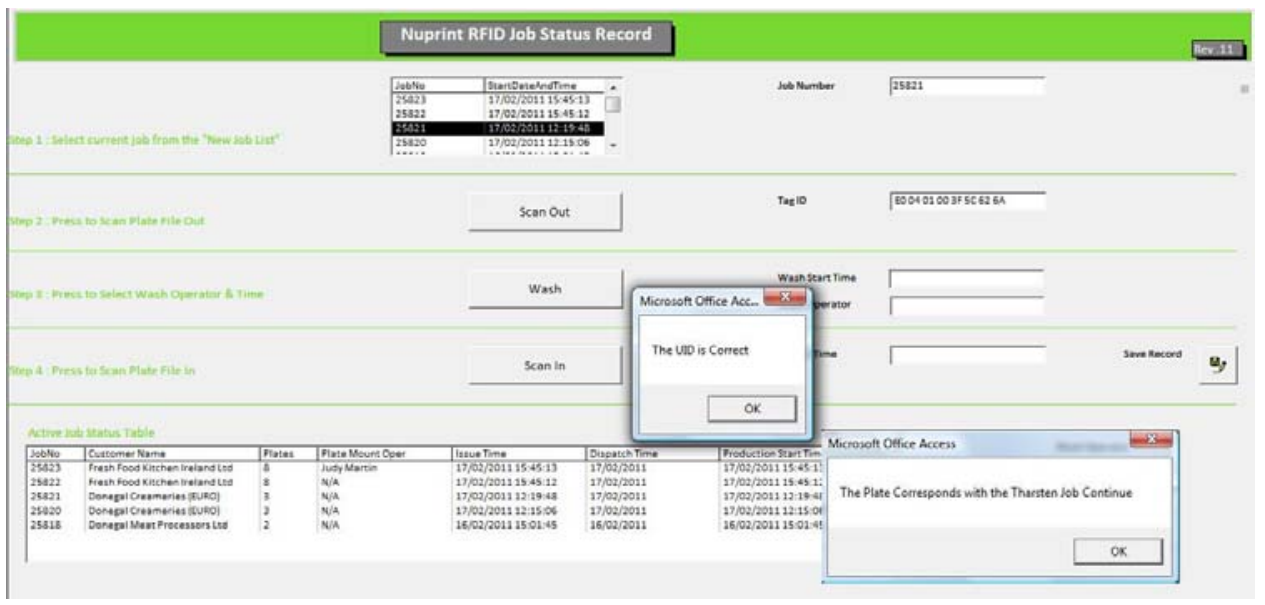

After Step 2 and confirmation that the correct UID has been selected the history results provided by Tharsten can now be made visible to the operative. The results appear at the bottom of the screen and inform the operative of the plate's history and any associated issues with them. The operative can then see from the previous comments that on Job number 25821 plate 3 was difficult to align, if this continues the operative may decide to purchase a new roll, they can also see that the plates have accumulated 5,949 meters in production, this also gives the operative an opportunity to order new plates in advance if they feel the plates have accumulated more than the average run time.

Step 3 is initiated after the job has finished in production and the operative is ready to wash down the used plates. The operative selects the 'Wash' button and the bound text boxes are populated with the last operative to sign into Tharsten and the associated time. Figure 18 shows the result.

Figure 18 Step 3 (see online version for colours)

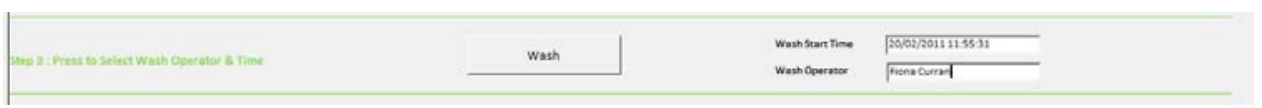

Step 4 is initiated after the plates have been successfully washed and are now ready to be stowed away. The operative selects the 'Scan In' button, a logical check is carried out to ensure the plate file that was scanned out is the one that is scanned in, if the result is correct the bound text box is populated with the current time. Figure 19 shows the result. The Job is now complete and the job number is removed from the active job list. 
Figure 19 Step 4 (see online version for colours)

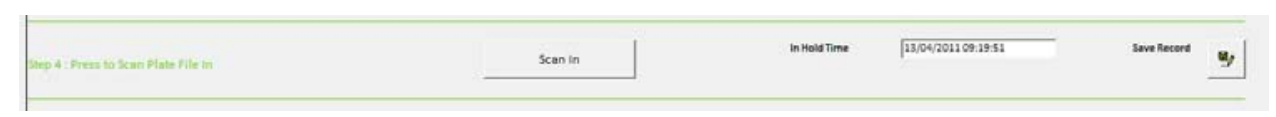

\section{System evaluation}

It is important to evaluate how this project actually delivers on the key target areas outlined at the start. Analysis during the baseline showed the area of greatest waste in the communications between areas, take place between the Preprep and production. Figure 20 shows the communication breakdown and the typical questions asked on a per job basis.

Figure 20 Detailed analysis of biggest concern (see online version for colours)

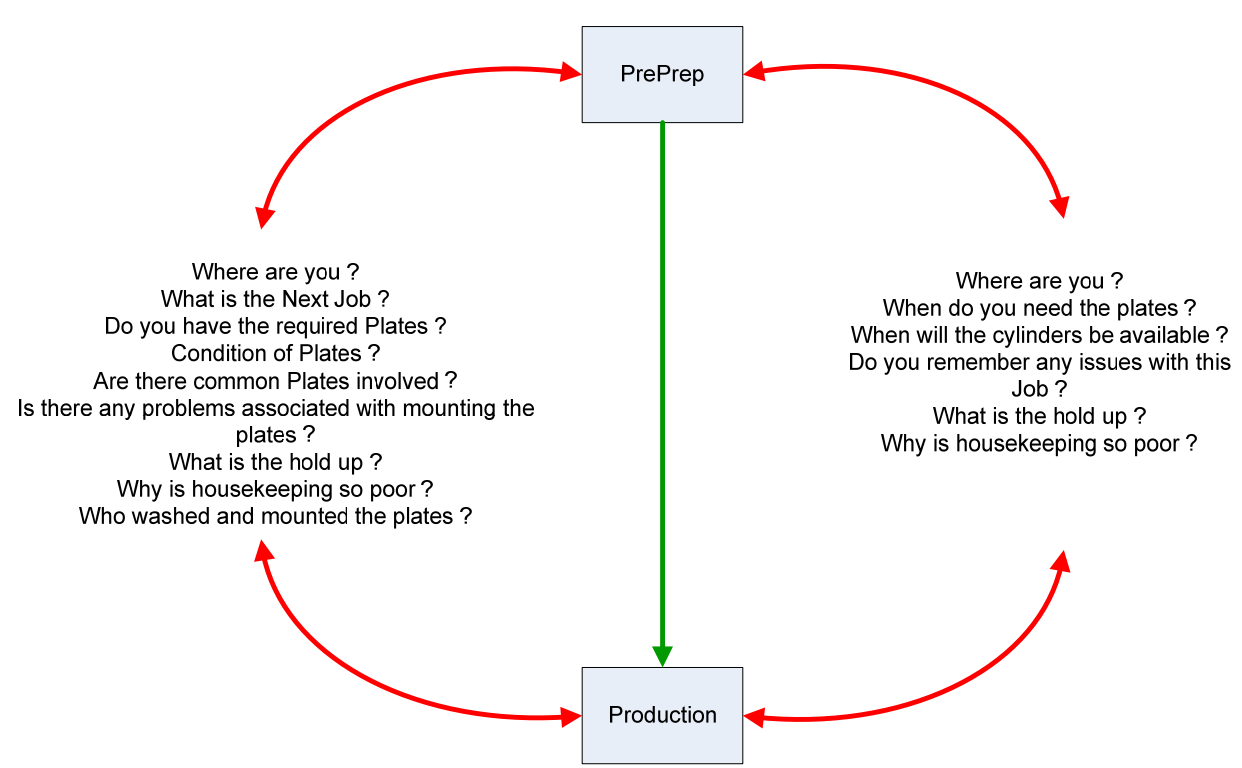

The implemented system will tackle these issues as outlined below:

1 Question 1: where are you?

- Implemented system counteraction: it is agreed that this question is asked in the majority as to ascertain the status of the plate files in the Preprep area, or, when they will be coming off the production line and therefore require cleaning. The systems interface when placed on the production floor will allow the production operative to see real time the Preprep operative removing the plates from storage and mounting them leaving them ready for collection by the production operative. Thus this communication will become obsolete. It may be the case however that the Preprep operative will still require communication off the Production operative as to when the job will be finished.

- System improvement: $50 \%$ 
2 Question 2: what is the next job?

- Implemented System Counteraction: the operatives both production and Preprep can clearly see from the interface the next job from Thasten (New Job List) and the next job to be issued to production (Active Job List).

- System improvement: $100 \%$

3 Question 3: do you have the required plates?

- Implemented system counteraction: the system will tackle this threefold, firstly in a proactively way whereby the operative must sign back in the plates thus cutting out the likely hood an operative will mislay the plates. Secondly production operative will not be required to ask this, he will simply see the plates have been mounted or not, thirdly the Preprep operative will have view to previous comment of missing plates or similar issues.

- $\quad$ System improvement: $70 \%$

4 Question 4: condition of plates?

- Implemented system counteraction: the system will allow comments to entered on a job basis and indicate the current condition of plates. The system also totals the accumulated meters produced by the plates giving the operative tangible information to judge the condition of the plates.

- System improvement: $100 \%$

5 Question 5: are common plates involved?

- Implemented system counteraction: the system at present does not deliver this function however this is a target for future enhancements.

- System improvement: $0 \%$

6 Question 6: is there any problems associated with mounting?

- Implemented system counteraction: the system will allow comments to be entered on a job by job basis and indicate any difficulties in the mounting of plates. In a bid to tackle the absurdity of a comments box a future enhancement may be to have a comment box for each area of concern i.e., plate condition, plate mounting issues etc whereby the operative is forced to populate using a drop down box instead of free text.

- System improvement: $80 \%$

7 Question 7: What is the hold up?

- Implemented system counteraction: the system will allow real time visibility of the Preprep area. Comments will allow descriptive text to be entered that can be made available to all clients informing them of the current situation or issues.

- $\quad$ System improvement: $80 \%$

8 Question 8: why is the housekeeping so poor?

- Implemented system counteraction: the single biggest cause for poor housekeeping in the Preprep area is plate files not being cleaned or stowed away. The system introduces an accountability factor whereby a named operative is undergoes the task of cleaning and storing the plate files this 
accountability should drive the housekeeping in a positive direction. The system will also highlight any plates that went out to production a suspiciously long time ago flagging any plates that may be left unattended, allowing management to ask questions before it becomes an issue.

- System improvement: $75 \%$

9 Question 9: who washed the plates?

- Implemented system counteraction: a system functional requirement is to associate an operative with the task of washing and cleaning the plates.

- System improvement: $100 \%$

10 Question 10: do you remember any issues with this job ?

- Implemented system counteraction: a system function is the ability to associate comments with a job or plate file that becomes viewable to the operative the next time the plate file is used.

- System improvement: $100 \%$

11 Question 11: When will the cylinders be available?

- Implemented system counteraction: the system at present does not deliver this function however this is a target for future enhancements.

- System improvement: $0 \%$

12 Question 12: When do you need the plates?

- Implemented system counteraction: the system gives the Preprep operative a view of what is being dispatched from Tharsten (i.e.,, New Job List). This naturally gives a prioritisation to the Preprep operative of first come first served. It is understood however in occasions late jobs will take priority which will have to be communicated directly.

- System improvement: $85 \%$

An overview of pre install waste communication is captured in Table 2, Table 6 shows this updated to reflect the savings made by the system post install. From Table 6 it can be seen that the time spent on overall communications is reduced from $37.1 \mathrm{~min}$ to $10.22 \mathrm{a}$ reduction in 26.88 minutes per job, which represents a $72.4 \%$ reduction

This data must now be equated to financial savings. From Table 6, we can see that the dispersion of the total saving26.88 minutes is broken down into 19.03min for Production and $7.85 \mathrm{~min}$ for Preprep. Assuming that Nuprint run 1.5 batches a day these times increase to 28.6 min for production and 11.8 for Preprep.

Taking a Preprep operative value at $£ 30.00$ per hour, the waste can be calculated as:

Financial waste $=($ Saved Time $(\min ) / 60) \times 30=(11.80 / 60) \times 30=£ 5.90$ per day

Taking a production operative value at $£ 120.00$ per hour, the waste can be calculated as:

Financial waste $=($ Saved Time $(\mathrm{min}) / 60) \times 120=(28.60 / 60) \times 120=£ 57.30$ per day

This equates to a total of $£ 63.10$ of financial saving per day. 
Table 6 Business evaluation

\begin{tabular}{lccc}
\hline Production to preprep & Actual time (pre) & \% Reduction & Actual time (post) \\
\hline Where are you & 4.4 & 50 & 2.2 \\
What is the next job & 2 & 100 & 0 \\
Do you have the required plates & 1.2 & 70 & 0.36 \\
Condition of plates & 2.6 & 100 & 0 \\
Are common plates involved & 0.5 & 0 & 0.5 \\
Problems associated with mounting the & 7.8 & 80 & 1.56 \\
plates & & & \\
What is holding you up & 1 & 80 & 0.2 \\
Why is the place so messy & 1 & 75 & 0.25 \\
Who washed and mounted plates & 3.6 & 100 & 0 \\
Section minutes wasted & 24.1 & & 19.03 \\
Section minutes saved & & & \\
Preprep to production & & & 2.2 \\
Where are you & 4.4 & 50 & 0.3 \\
When do you need the plates & 2 & 85 & 2.2 \\
When will the cylinders be available & 2.2 & 0 & 0.2 \\
Do you remember issues with job & 2.4 & 100 & 0.2 \\
What's holding you up & 1 & 80 & 0.25 \\
Why is the place so messy & 1 & 75 & \\
Section minutes wasted & 13 & & \\
Section minutes saved & & & \\
Actual time taken pre solution installation & 37.1 & & \\
Actual time taken pre solution installation & & & \\
\hline & & & \\
\hline
\end{tabular}

As well as the everyday savings there is also non-regular occurrences highlighted during base lining that the system tackles these types of individual issues are as follows.

- Individual issues: a typical example of an individual issue was, during a period when the pre-prep operator was off ill the pre-prep operators duties fell on the production operators. However, the process of washing the plates is an undesired task coupled with the limited time the production operator had, it was decided the plates would be left unwashed in a pile and not cleaned or filed away. The consequence of this was that when an urgent job came through, a delay was incurred in locating the plates. When the plates were located a further delay was incurred because they had to be washed and mounted. Once the plates were placed on the machine and the job 'setup' ready for 'signoff', it transpired that two were damaged as they had been situated at the bottom of the pile and were subsequently compressed. This necessitated the re-purchase of two $£ 70$ plates at a $£ 10$ delivery cost. The operations control was also faced with the dilemma of cleaning down the machine to run a job while the replacement plates were being manufactured and delivered or to hold off on the wash-up / setup and run the job the next day. This expense was in addition to delaying delivery of the labels. The result of this bad relationship was an escapade 
wasting £100's and damaged reputation with a customer. After site consultation this likelihood and financial waste was evaluated at $£ 250$ per month, i.e., approximately $£ 12.5$ per day.

To summarise the system is saving approximately $£ 63.10+£ 12.50=£ 75.60$ per day. This saving is substantial when equated to yearly savings of $£ 18,144.00$ calculated at 5 day week, 4 weeks per month and 12 months per year. Not only is the saving justification enough but throughout this project it has become clear that the companies' ability to introduce and work with RFID technology is of utmost importance. RFID is becoming a technology that more and more label purchasers are requesting to fulfil their own manufacturing processes. Having this technology already in the company's portfolio allows them to be proactive in selling the technology in the market place and not be driven to it by customers who could go elsewhere in this competitive market space.

\section{Conclusions}

RFID systems are becoming more and more embedded in the supply chain. Tracking goods in the work place has been identified as one area where production can be improved. RFID technology can be used in different manufacturing scenarios such as being attached to items that are moving through the factory on conveyer belts or being moved around by staff on trucks or forklifts. As the tags move around the factory floor or the warehouse they pass by readers and the tags can be then tracked using intelligent software and database application. In modern manufacturing facilities, there are work flow process systems that can be improved to reduce the overall work load and increase the total work throughput. Lean manufacturing can provide beneficial results for manufacturing facilities but only based on extensive, accurate data. This project initially assessed a manufacturing plants work flow processes and evaluated them against industry best practices. RFID was identified as a technology which could help stream line the flow of work on the factory floor. It was clear initially that there were NVAT in the system. However obvious this waste is to anyone that analyses the system, it was of utmost importance to numerically evaluate this waste. This numerical evaluation base line, in terms of finance and time, will firstly keep focus on the project aims throughout the period of the project, and secondly allow for proper evaluation and justification for the end install. The design and implementation sections outline the system developed. After consultation with Nuprint it was clear that they required two main interfaces, one, to maintain their plate file database allowing the RFID labels to take over the old labelling system of Plate File numbers. The second interface is to allow production information to be inputted for the beginning and end of a job cycle in the Preprep area. These interfaces are unambiguous, and simple to use. They do not increase the work load of operations and whether gathering information from the existing MIS system, RFID or Access, it appears to be one coherent system. An area which is now being explored is to introduce QR codes which can work with handheld Android devices.

Ultimately, the system provides a mass amount of information to the system and allows proper analysis of how the system operates and how it can be modified to enhance KPI's. This information input not only provides great transparency between departments but allows management to oversee the complete process, and answer questions such as whether any jobs that went for production are not returned to storage, the length it 
normally takes for job A to be completed, and whether areas such as pre-prep hold up production or vice-versa? The main aim was to provide a solution that reduces waste in the company. The key here was not just the RFID technology but rather the data manipulation in the MES which must be capable of providing transparent accurate, easy to access data to all departments. It was found that this system will save approximately $£ 63.10+£ 12.50=£ 75.60$ per day. This saving is substantial when equated to yearly savings of $£ 18,144.00$ calculated at five day week, four weeks per month and 12 months per year. Throughout this project it became clear that companies' ability to introduce and work with RFID technology is of utmost importance. RFID is becoming a technology that more and more label purchasers are requesting to fulfil their own manufacturing processes. Having this technology already in companies' portfolio allows them to be proactive in selling the technology in the market place and not be driven to it by customers who could go elsewhere in this competitive market space.

\section{References}

Chen, X., Xie, Z.X. and Zheng, L. (2009) 'RFID-based manufacturing execution system for intelligent operations', 16th International Conference on Industrial Engineering and Engineering Management, 21-23 October, pp.16-24, Dresden, Germany.

He, W., Tan, E., Lee, E. and Li, T. (2009) 'A solution for integrated track and trace in supply chain based on RFID \& GPS', IEEE ETFA 2009 Conference on Emerging Technologies \& Factory Automation, 22-26 September, pp.56-64, Mallorca, Spain.

Huang, G., Yuan, G. and Li, J. (2010) Developing Real-Time Manufacturing Execution System for Automobile Assembly Factory, Intelligent Control and Information Processing (ICICIP), 13-15 August, pp.696-700, Wuhan, China.

Lahiri, S. (2005) RFID System in RFID Sourcebook, New York, USA, ISBN: 978-953-7619-73-2.

New, S.J. (2007) 'Special issue: the Toyota production system: thirty years of research, and beyond', International Journal of Production Research, Vol. 45, No. 16, pp.3545-3778.

Ohashi, K., Ota, S., Ohno-Machado, L. and Tanaka, H. (2008) 'Comparison of RFID systems for tracking clinical interventions at the bedside', AMIA Annual Symposium Proc., pp.525-529, Hawaii, USA.

Ritsma, R.J., Tuyl, A. and Snijders, B. (2009) 'Buying the lowest Total Cost of Ownership (TCO)', PCIC Europe, 21-24 June, pp106-112, Barcelona, Spain.

Willhite, J. (2004) 'Implementing the principles of lean manufacturing at Semicon Associates Samarium Cobalt Magnet Facility', IVEC 200 - Fifth IEEE International on Vacuum Electronics Conference, 27-29 April, pp104-105, London, UK.

Womack, J., Jones, D. and Roos, D. (1990) The Rise of Lean Production in The Machine That Changed the World, London, UK, ISBN: 9780743299794.

Wong, Y., Wong, K.W. and Ali, A. (2009) 'Key practice areas of lean manufacturing, International Association of Computer Science and Information Technology - Spring Conference, 2009.IACSITSC '09, pp.82-90, New York, USA. 